Article

\title{
Key Technologies in the Context of Future Networks: Operational and Management Requirements
}

\author{
Lorena Isabel Barona López ${ }^{\dagger}$, Ángel Leonardo Valdivieso Caraguay ${ }^{\dagger}$, \\ Marco Antonio Sotelo Monge ${ }^{\dagger}$ and Luis Javier García Villalba ${ }^{*}+$ \\ Group of Analysis, Security and Systems (GASS), Department of Software Engineering and Artificial \\ Intelligence (DISIA), Faculty of Computer Science and Engineering, Office 431, Universidad Complutense de \\ Madrid (UCM), Calle Profesor José García Santesmases , 9, Ciudad Universitaria, 28040 Madrid, Spain; \\ lorebaro@ucm.es (L.I.B.L.); angevald@ucm.es (Á.L.V.C.); masotelo@ucm.es (M.A.S.M.) \\ * Correspondence: javiergv@fdi.ucm.es; Tel.: +34-91-394-7638 \\ + These authors contributed equally to this work.
}

Academic Editor: Dino Giuli

Received: 11 September 2016; Accepted: 7 December 2016; Published: 22 December 2016

\begin{abstract}
The concept of Future Networks is based on the premise that current infrastructures require enhanced control, service customization, self-organization and self-management capabilities to meet the new needs in a connected society, especially of mobile users. In order to provide a high-performance mobile system, three main fields must be improved: radio, network, and operation and management. In particular, operation and management capabilities are intended to enable business agility and operational sustainability, where the addition of new services does not imply an excessive increase in capital or operational expenditures. In this context, a set of key-enabled technologies have emerged in order to aid in this field. Concepts such as Software Defined Network (SDN), Network Function Virtualization (NFV) and Self-Organized Networks (SON) are pushing traditional systems towards the next 5G network generation. This paper presents an overview of the current status of these promising technologies and ongoing works to fulfill the operational and management requirements of mobile infrastructures. This work also details the use cases and the challenges, taking into account not only SDN, NFV, cloud computing and SON but also other paradigms.
\end{abstract}

Keywords: 5G; future network; NFV; SDN

\section{Introduction}

The emergence of a new business model and services (e-solutions, e-health, e-commerce, Voz IP, streaming, among others) and the exponential growth in the information circulating on the Internet has brought unexpected challenges to the IT industry. The development of new infrastructures, known as Future Networks, is focused on ensuring robustness, security, scalability and the fast deployment of applications through the customization of network behavior.

According to the Future Internet 2020 Report of the European Commission, the development of a new generation of networks takes an average of 10 years, this means that the concept of Future Networks is coming soon. Future Networks must provide a flexible, reliable, secure, smart and high-performance environment to connect the digital society, while leveraging the competitiveness, faster innovation and standardization of new technologies. This network must embrace not only current services but also any kind of elements (Internet of Things-IoT) [1]. These kind of networks will generate a significant impact not only on the societal but also on the operational field. On one hand, Future Networks must cover the necessities of smart cities, entertainment, public security, etc., providing a wide range of network services and applications [2]. Users will expect 
enhanced Quality of Experience (QoE) with minimal disruptions of the services, regardless of their location, the kind of device, or when the service is required. On the other hand, Future Networks will help to decrease the capital and operational expenditures (capex/opex) related to the deployment and management of new applications and infrastructures with substantially reduced service creation time [3]. Nowadays, the introduction of novel technologies is a time-consuming process due the slow standardization process, manual service deployment or the semi-automated management tasks. In the context of mobile networks, the average revenue per user (ARPU) is continuously decreasing, while the demand on mobile traffic keeps growing. This causes a negative response by network operators to invest in new network hardware infrastructure. In order to lay the foundations of Future Mobile Networks, three fields must be improved: Radio, Network and Operations and Management capabilities [4].

- Radio capabilities leverage the spectrum optimization, enhance interference coordination mechanisms and support dynamic radio topologies through the exploitation of higher frequencies, enabling cost-effective dense deployments, intelligent and dynamic coordination of multi Radio Access Technology (RAT), as well as sharing resources, among others.

- Network functionalities will enable the creation of an open environment in order to support several use cases in a cost-effective manner by means of the enhancement of user devices, minimizing the number of deployed entities and splitting the control and user plane functions (open its interfaces).

- The operation and management capabilities are intended to simplify operations not only in network control tasks but also in the deployment of new services, without increasing the system complexity. This field also includes reactive and proactive mechanisms to enhance the decision-making in control and management operations. This characteristic will enable the deployment of virtualized components, wherever they might be needed.

Radio and Network capabilities are topics well-studied in the literature [5-8]. In [6], a detailed survey and ongoing projects related to $5 \mathrm{G}$ networks are presented. This work discusses some emerging technologies, such as massive Multiple Input Multiple Output (MIMO), cognitive radio, cloud technologies and Device to Device Communication (D2D) in order to tackle the following requirements: enhanced data rate, spectral efficiency, lower latency, deployment and management of ultra dense networks. For their part, Boccardi et al. [5] describe five disruptive concepts that might impact on the development of 5G Radio requirements. They take into account the ability for devices to communicate between themselves (Machine to Machine communication-M2M), spectrum and resource optimization (massive MIMO and millimeter wave), the introduction of a device-centric concept and smarter devices (allowed to play an active role in the network). Regarding Network capabilities, one of the main challenges is to create an open, multi-tenant and service-oriented environment to support large amounts of traffic while covering different kinds of Quality of Service (QoS) levels and Service Level Agreements (SLA), in terms of latency, bandwidth or jitter. This environment will allow a flexible reconfiguration of network devices and programmability features based on the device-level, application, user and environment context [7]. Meanwhile, the introduction of intelligence in 5G systems might enable the improvement of the resource use (spectrum, transmission power levels and other radio resources), cost-effective energy mechanisms and flexible cell management (different sizes) [9].

In order to tackle operation and management capabilities and enable ubiquitous connectivity, the research community proposes the introduction of some key technologies, such as SDN [10], NFV [11], Cloud Computing [12], Self-Organized Network (SON) [13] and Machine Learning [14]. $\mathrm{SDN}$ is based on the separation of the control plane from the data plane in traditional network devices. This decomposition allows the centralized control of the network with greater automation capacities and it simplifies the management process. For its part, NFV allows the implementation of traditional Network Functions (NF) as virtualized instances, running in a generic hardware. The main advantage 
of NFV is its improved scalability capacity which, due to Virtual Network Functions (VNF), can be deployed anytime and anywhere in minutes, whereas previously it took more time (compared with traditional functions) [15]. From the technical point of view, SDN and NFV are complementary technologies, and together could facilitate configuration and network customization [16]. For their part, concepts such as Cloud Computing and SON allow the easy deployment of services (on-demand fashion) and enhanced traffic management based on intelligence decisions.

SDN, NFV, Cloud computing and SON are enablers that provide business agility and simplify the operation and management tasks. In contrast to traditional mobile systems, future networks will enable operators to control the traffic information (via SDN) in order to use only necessary network functions in a shared virtualized network (NFV and cloud computing). These technologies also allow the reduction of the complexity of planning, configuration and optimization tasks in the whole system, giving the capability to reuse existing infrastructures in a proactive way.

The main objective of this paper is to present a full view of the applicability of these technologies as well as the finished and ongoing projects in order to cover the operational and management requirements of the future mobile networks. It also provides the current use cases that will leverage the development of services. This paper is organized as follows. Section 2 presents the key-enabled technologies that could be taken into account for the design of future mobile networks. Section 3 presents an overview of the main research, projects and use cases that are based on these technologies. Then, Section 4 describes the challenges and future trends related to the adoption of these technologies and opens a discussion about the future of mobile networks. Finally, the conclusions of this work are presented in Section 5.

\section{Key-Enabled Technologies for Future Networks}

Future Networks envisage a fully connected society where the user can enjoy enhanced services and the operators obtain enough revenues from the deployment and provision of their services. In this context, Future Networks could benefit from the evolution of novel technologies such as SDN, NFV, $\mathrm{SON}$ and Cloud Computing, as is detailed below.

\subsection{Software Defined Networking}

Software Defined Networking $[10,17]$ is a novel architecture in network communications. SDN proposes the separation between data and control planes in network devices and a centralized control of the network. In SDN, three well defined layers are established: infrastructure layer, control layer and application layer Figure 1.
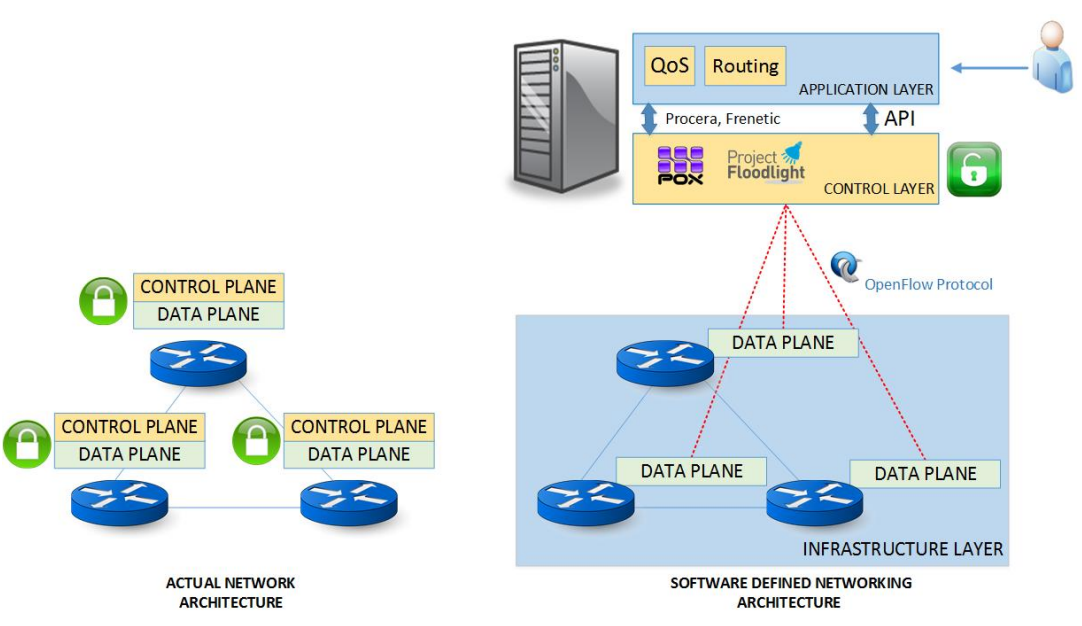

Figure 1. SDN vs. Traditional Architecture. 
Infrastructure layer (data plane) refers to the physical hardware and/or basic software components of the network devices responsible for sending the packets through the network. In other words, this component does not make logical decisions about the network behavior. For its part, the control plane analyzes the information received by the data plane and makes decisions about the network behavior. The control plane offers functionalities to the application layer, which uses these functions to establish high level network policies or services. To connect hardware devices with a SDN controller, a standardized protocol is needed. In practice, OpenFlow is the first SDN Open Protocol initiative adopted by the research community [18]. OpenFlow uses the most common hardware capabilities of network devices (flow tables) and opens those up. With this approach, the whole network behavior can be controlled and dynamically adapted according to user needs. Figure 1 shows the principal differences between traditional and the SDN architectures and their components. It is important to note that SDN also eliminates the complexity and the closed nature of traditional networks. Future Networks can take advantage of the main characteristics of SDN in order to enhance the control, management and customization of the network services. In the context of mobile networks, SDN will allow the management of mobile resources in an easy manner, providing a better QoS in an open environment.

\subsection{Network Function Virtualization}

The concept of virtualization has allowed resource sharing (e.g., hardware) between different tenants, each one with its private functionality (e.g., different Operating System). For its part, network virtualization enables the sharing of network resources with different virtual topologies and forwarding logic in the same network infrastructure (e.g., Virtual Local Area Network-VLAN) [15]. However, if a service provider needs a specialized network application (firewalls, Deep Packet Inspection-DPI, etc), it must add a new hardware device. It represents an extra investment (capex/opex) and creates scalability and innovation constraints. In this context, the NFV has gained importance between network operators for its facilities to deploy services or applications as software network functions that can be automatically instantiated in different parts of the infrastructure. In 2012, main telecommunication service providers and the European Telecommunications Standards Institute (ETSI) proposed the NFV approach [11]. This allows the easy deployment of network functions on standard switches, storage or high volume servers as shown in Figure 2.

The referential architecture defines three main components: the Network Function Virtualization Infrastructure (NFVI); VNFs; and NFV Management \& Orchestration (NFV M\&O). NFVI controls the hardware resources (computing, storage and network), and uses a virtualization process in order to create software instances (virtual compute, virtual storage and virtual network). VNFs are the network functions that run over the NFVI. NFV M\&O orchestrates and manages VNFs and the NFVI. Additionally, this module works with the external Business Support System and Operation Support System (OSS/BSS) in order to bill the services. The main objective of this model is to provide an architecture that allows the easy deployment, orchestration and management of traditional network functions as virtualized instances. These characteristics will enable future infrastructures to provide services regardless of the type of devices or their location and will promote on-demand service modality. 


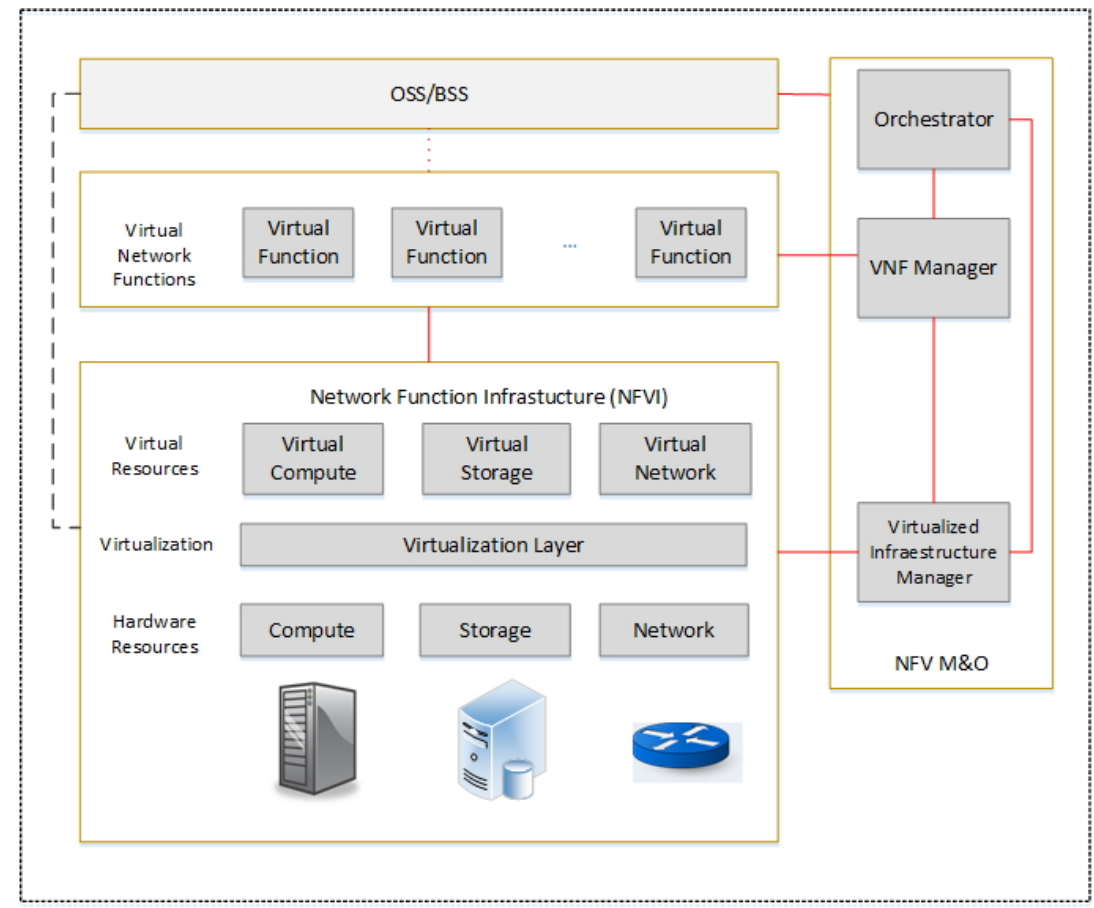

Figure 2. NFV Reference Architecture.

\subsection{Cloud Computing}

With the rapid evolution of the services, processes and enterprise business models, traditional provisioning of resources (computing, storage and network) has evolved to a new concept known as Cloud Computing [12]. In cloud computing, a particular resource can be leased by users to a third party, according to their needs. This innovative service-oriented model provides an elastic infrastructure, while decreasing the costs of hardware acquisition. Cloud computing offers different service models depending on the available resources to be leased. For instance, Software as a Service (SaaS) enables the sharing of software programs running on a cloud infrastructure. For its part, Platform as a Service (PaaS) architectures allow users to execute customized programs created with different libraries, tools, or programming languages supported by the cloud environment. In the Infrastructure as a Service (IaaS) model, the user is able to customize the different computing resources (processing, storage, and network) and deploy arbitrary software (e.g., different Operating Systems). In particular, Openstack [19] appears as a promising open source project to manage cloud platforms through its set of services (nova, neutron, keystone, telemetry, etc.), which could be integrated not only into traditional networks, but also SDN and NFV approaches. In the context of Future Networks, cloud computing would facilitate on-demand network access to available resources by means of its elastic capacity. This modality benefits the final users as well as the service provider.

\subsection{Self-Organized Networks}

The exponential increase of on-line services (e-bank, e-health, streaming) and the number of connected devices has brought new challenges to the network infrastructure in terms of security, performance and reliability. The management and rapid response to unexpected problems in the network (link failure, congestion, Distributed Denial of Service-DDoS, delay) is fundamental to guarantee QoS/QoE to users. Network intelligence mechanisms are needed in order to resolve/mitigate possible problems, to decrease the service recovery time and the operational costs [14]. Moreover, the use of advanced techniques such as artificial intelligence, data mining or pattern recognition enables proactive and reactive self-management actions capable of preventing potential problems and maintaining the subscribed network services. However, the implementation of 
self-organized solutions [13] in current networks is limited by the rigidity of the traditional network architectures. The modification or customization of a flow in the network requires the individual configuration of each network device, and the deployment of new network services/protocols, from design to implementation, can take a long time. In this context, the smart integration of the novel technologies listed above has the potential to provide operators a smart network infrastructure capable of managing complex network scenarios and reducing operational costs, as shown in Figure 3.

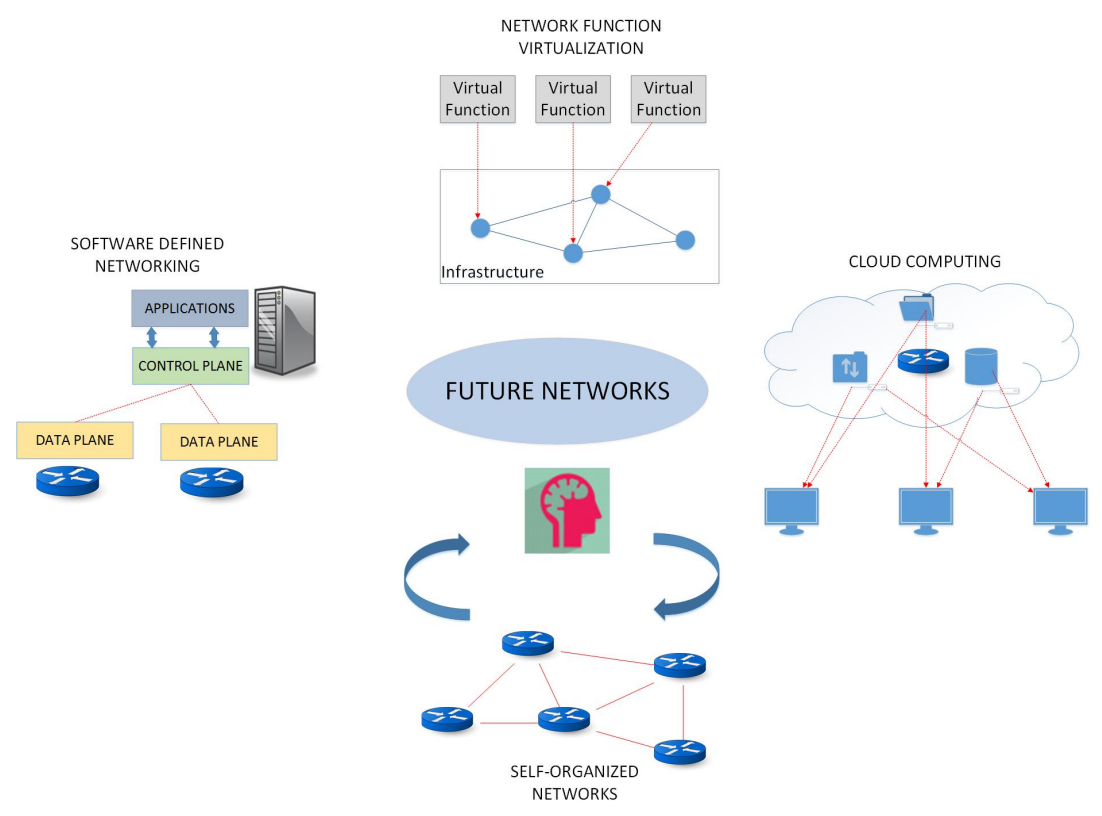

Figure 3. Trends in Future Networks.

The combination of SDN with NFV aims to enhance the management and orchestration process in current networks. On one hand, SDN can control the network behavior and it may require specific network functions in order to fulfill the new requirements and ensure the QoS levels established by the service provider. On the other hand, NFV allows the rapid deployment of these functions, without constraints of location or hardware vendor. For its part, cloud computing and SON could facilitate the scalability of the services, the customization of network infrastructure and the smart control and management of the available resources. It is expected that machine learning and data mining can provide higher and more intelligent mechanisms related to monitoring and management tasks [14]. It is important to note that future mobile networks envisage to provide a system driven by software, relying on technologies such as SDN, NFV, cloud computing and SON. These concepts aim to cover flexibility needs in terms of resource allocation, smarter monitoring and the introduction of new network functions, while ensuring the quality of the services. Furthermore, these technologies have been applied in radio and network fields in order to tackle their requirements.

\section{Research Overview}

Future Mobile Networks require customizable, efficient and scalable network infrastructures in order to meet the new user needs and the exponentially-increasing traffic demands, while decreasing the capital and operational expenditures. The SDN concept has been introduced in a broad range of fields, such as QoS, data centers, mobile and optical networks, security, network virtualization, among others [17]. As an instance, Google was one of the first enterprises to incorporate the SDN concept to communicate their internal Datacenter-WAN. Furthermore, there are some projects that allow SDN experimentation by offering scalable testbed infrastructures with research purposes, such as Geant, GENI, Ofelia, Felix, among others [20]. 
In particular, the integration of SDN or NFV with mobile networks includes the deployment of virtualized base stations and core components (Long Term Evolution-LTE) [21], energy efficiency experimentation on WiFi networks, the optimization of very dense and heterogeneous wireless networks [22], etc. The next generation of mobile networks could take advantage of the combination of key-enabled technologies to enhance the following areas: (i) the development of radio access (high speed, spectrum efficiency, high mobility, high availability); (ii) improvements in core networks (QoS support, aggregated processes, network slicing, cloud deployment) and (iii) the management and orchestration process (customization of user needs, dynamic allocation of resources, energy efficiency mechanism, manage a big amount of data) $[5,9,23]$.

Different standard organizations leverage the adoption of SDN and NFV concepts in their infrastructures. These organizations have presented the challenges, Key Performance Indicators (KPI) and possible use cases in order to cover the above-mentioned areas. As an instance, Open Networking Foundation (ONF) [24] promotes the adoption of SDN and defines a wide range of use cases, such as inter-cell interference management, virtual customer edge, network virtualization or data center optimization. Meanwhile, NFV is an initiative of ETSI and telecommunication providers, which proposes the virtualization of the traditional network functions. ETSI-NFV defines nine general use cases [25], such as NFV Infrastructure as a Service (NFVIaaS), VNF Forwarding Graph (VNF-FG), etc. In the scope of mobile networks, NFV promotes the virtualization of Mobile Core Networks and IP Multimedia Subsystem (IMS), the virtualization of a mobile base station, the virtualization of the home environment and the virtualization of Content Delivery Networks (CDNs). In the meantime, some open source projects led by the research community have emerged to provide an open environment to test with SDN, NFV and cloud computing, such as OpenNFV (SDN and NFV) [26], Floodlight (OpenFlow and OpenStack support) [27], OpenDaylight (SDN, NFV and OpenStack) [28], among others.

Regarding mobile networks, industry manufacturers, telecommunication operators, and related stakeholders are working on the definition of requirements, standardization, regulation and development of future mobile systems, such as 5G-PPP (5G Infrastructure Public Private Partnership) and Next Generation Mobile Network initiative (NGMN). The 5G-PPP [29] proposes solutions, standards and infrastructures to allow the ubiquitous 5G communication. For its part, the NGMN [4] will expect to provide $5 \mathrm{G}$ solutions by 2020, within eight general use cases: broadband access in dense areas, broadband access everywhere, high user mobility, massive Internet of Things, extreme real-time communication, lifeline communication, ultra-reliable communication and broadcast-like services.

The most outstanding efforts have been made in the 5G research field. A wide range of projects or initiatives will expect to cover the needs of future mobile users. These worldwide initiatives encompass global regions of Asia, Europe and the Americas.

With the aim to promote the adoption of 5G in Asia, China has launched the IMT-2020 promotion group [30], which manages five working groups: Requirements, Technology, Spectrum, Intellectual Property Right (IPR) and Standardization. This is the most important promotion platform related with research and international cooperation purposes. Similarly, coordinated efforts in the 5G area have been launched in South Korea and Japan, the former with the 5G Forum [31] and the latter with the Fifth Generation Mobile Communications Promotion Forum (5GMF) [32]. Both are conducting research projects involving active participants from the government, industry, and academia, in order to facilitate the development of 5G.

Significant efforts have been made in Europe under the support of the European Union Framework Project 7 (FP7) and the Horizon 2020 programmes [29]. On the one hand, FP7 has launched 5G research projects such as METIS, MCN, CONTENT, T-NOVA, UNIFY, CROWD, etc. On the other hand, Horizon 2020 has financed several research projects (considered by 5G PPP as Phase 1 Projects) such as 5G-NORMA, METIS II, CHARISMA, SONATA, FLEX5GWARE, SELFNET, among others.

In the FP7 context, Mobile and wireless communications Enablers for the Twenty-twenty Information Society Project (METIS) [33] lays the foundations of 5G networks and promotes the general agreement to design this mobile environment. The first phase of this project (METIS I) includes five big 
scenarios (amazingly fast, great Services in a crowd, ubiquitous things communicating, best experience follows you, super real-time and reliable connections) in a use-case driven approach.

The initiative Mobile Cloud Computing (MCN) [34] provides mobile services by means of the combination of three components: mobile network, compute, and storage resources. MCN defines a wide range of use cases, such as Radio Access Network (RAN) on Demand, Mobile Virtual Resources on Demand, Machine Type Communication on Demand, SDN or virtualized Evolved Packet Core (EPC), to mention a few. In the same way, the CONTENT project [35] proposes a network infrastructure that enables end-to-end cloud and mobile services. This project provides a virtualized infrastructure based on LTE, WIFI and optical metro networks and introduce the SDN concept in their deployment. CONTENT presents two general use cases: Infrastructure and network sharing (created logical resources) and cloud service provisioning on top of virtual infrastructures (end-to-end).

The integration of SDN with NFV is proposed in T-NOVA [36] and UNIFY [37] projects. On one hand, T-NOVA provides a framework to deploy VNFs over network infrastructures. The innovation of this project consists of their NFV Apps marketplace, which enables the easy creation, deployment and management of virtual network appliances in a standardized environment. T-NOVA proposes three general scenarios: High-Level Scenario, T-NOVA VNFs, and VNF Chaining. On the other hand, the UNIFY project takes advantage of cloud computing and the virtualization concept to provide a novel network architecture with optimized data traffic flows and the dynamic placement of networking, computer and storage components. This project presents eleven use cases, organized around the following domains: Infrastructure Virtualization, Flexible Service Chaining and Network Service Chain Invocation for Providers.

In the area of SDN and SON, CROWD [22] includes these technologies to enhance the coordination process between radio base stations in very dense and heterogeneous wireless networks (Dense Nets). This project allows the network cooperation, the dynamic network configuration, dynamic backhaul reconfiguration, energy optimization, etc. CROWD also presents fifteen use cases divided into two big scenarios: self-optimising dense networks and Optimised mobility in dense radio access networks. As part of the Horizon 2020 programme, 5G-NORMA [38] is a research project which aimed to provision an adaptive and open 5G infrastructure with capabilities to service customization, enhanced performance and security. To this purpose, this project introduces adaptability capacity to allocate mobile network functions in the most appropriate location and in a short time. Likewise, METIS II [39] presents a novel 5G RAN design, introducing a protocol stack architecture intended to provide a seamless integration of $5 \mathrm{G}$ radio technologies. The innovations of METIS II are focused on the spectrum management, air interfaces harmonization, resource management and a common control and user plane framework. The integration of them will support regulatory and standardization bodies. Other ongoing H2020 projects that combine SDN and NFV technologies are CHARISMA [40] and SONATA [41]. CHARISMA will enable the deployment of an intelligent cloud radio access network (C-RAN) and virtualized Customer Premise Equipment (CPE). SONATA will support network function chaining and an enhanced orchestration process in order to allow service customization.

The provision of innovative hardware and software platforms to support $5 \mathrm{G}$ infrastructures is proposed in FLEX5GWARE [42]. This project attempts to develop and prototype key components of 5G networks in the hardware and software domains. The main objective of this project is to deliver a highly reconfigurable hardware platform together with a well suited software platform, over which network elements and devices can be deployed following a modular, efficient and scalable approach. Several components must be deployed as 5G enablers, such as MIMO emulators, high-speed broadband converters, Filter Bank Multi-Carrier (FBMC) transceivers, Low-Density Parity Check (LDPC) codes, etc., with suitable interfaces to allow flexible software-based management schemes.

The integration of SDN, SON, NFV and Artificial Intelligence is encompassed by the SELFNET $[43,44]$ project, which introduces intelligent, self-organizing and autonomic capacities to $5 \mathrm{G}$ networks, taking advantage not only of SDN and SON but also NFV and Cloud Computing. This project will provide a scalable, extensible and smart architecture to foster innovation and decrease 
capital and operational expenditures derived from network management tasks. Moreover, SELFNET introduces the SON concept to facilitate the automatic management of network infrastructures. SON solutions are typically classified into three domains: self-protection, self-optimization and self-healing, which are the use cases proposed by SELFNET. Likewise, the COGNET Project [45] proposes the introduction of machine Learning, SDN and NFV in order to enhance monitoring tasks and autonomic network management. COGNET predicts the resource demand requirements and then changes its own configuration based on the network analysis (prediction, frauds, detecting error and security conditions).

Table 1 shows the current European use-case driven projects that tackle different $5 \mathrm{G}$ requirements, through a combination of SDN, NFV, SON and cloud computing concepts. All of these projects take into account SDN in different domains, such as e-health services, security, service chaining, multimedia optimization, etc.

Table 1. Research Projects in Mobile Networks.

\begin{tabular}{|c|c|c|c|}
\hline Project Name & Related Technologies & Main Objective & Scenarios/Use Cases \\
\hline MCN [34] & $\begin{array}{l}\text { - SDN } \\
\text { - Cloud Computing }\end{array}$ & $\begin{array}{l}\text { Enhanced traffic processing by means of } \\
\text { the separation between radio hardware } \\
\text { and packet forwarding hardware. }\end{array}$ & $\begin{array}{l}\text { - Cloud Computing for Mobile } \\
\text { Network Operations } \\
\text { - End-To-End Mobile Cloud }\end{array}$ \\
\hline UNIFY [37] & $\begin{array}{l}\text { - } \mathrm{SDN} \\
\text { - NFV }\end{array}$ & $\begin{array}{l}\text { The development of an automated and } \\
\text { dynamic service provision platform, } \\
\text { based on a service chaining architecture }\end{array}$ & $\begin{array}{l}\text { - Infrastructure Virtualization } \\
\text { - Flexible Service Chaining } \\
\text { - Network Service Chain } \\
\text { Invocation for Providers }\end{array}$ \\
\hline CROWD [22] & $\begin{array}{l}\text { - SDN } \\
\text { - SON }\end{array}$ & $\begin{array}{l}\text { The creation of technologies to support } \\
\text { dynamic network functionality } \\
\text { configuration and fine, on-demand, } \\
\text { capacity tuning. }\end{array}$ & General scenario \\
\hline 5G-NORMA [38] & $\begin{array}{l}\text { - SDN } \\
\text { - NFV }\end{array}$ & $\begin{array}{l}\text { The development of an adaptive, } \\
\text { customizable, secure and efficient } \\
\text { mobile network architecture to deal } \\
\text { with complex traffic } \\
\text { demand fluctuations. }\end{array}$ & $\begin{array}{l}\text { - Multi-service } \\
\text { - Multi-tenancy }\end{array}$ \\
\hline COGNET [45] & $\begin{array}{l}\text { - } \mathrm{SDN} \\
\text { - } \mathrm{NFV} \\
\text { - } \text { Machine Learning }\end{array}$ & $\begin{array}{l}\text { Dynamic adaptation of the network } \\
\text { resources (virtual network functions), } \\
\text { while minimizing performance } \\
\text { degradations and fulfill SLA } \\
\text { requirements. }\end{array}$ & $\begin{array}{l}\text { - Situational Context } \\
\text { - Just-in-time Services } \\
\text { - User-Centric Services } \\
\text { - Optimized Services } \\
\text { - SLA Enforcement } \\
\text { - Collaborative Resource Manage }\end{array}$ \\
\hline
\end{tabular}


Last, but not least, it is worth mentioning the research efforts in the Americas, where a group of telecommunication service providers and manufacturers created the 5G Americas [46], an organization intended to foster the development of LTE wireless technology leveraging the adoption of 5G in the North and South America's society. At the same time, several activities have been conducted by academia. For instance, the Berkeley Wireless Research Center (BWRC) involves university, industry, government and other research stakeholders focused on exploring innovations in wireless communication systems based on radio frequency and millimeter wave technologies, which are its main challenge to develop reconfigurable radio architectures. Likewise, the Broadband Wireless Access and Applications Center (BWAC) involves around fifty research centers with the aim to collaborate with the industry in the creation of innovative and scalable wireless networks.

\section{Future Trends and Challenges}

The current necessities address the direction of the business and the requirements of Future Networks. It is expected that $5 \mathrm{G}$ networks will cover the increase of traffic volume by means of improving spectrum utilization, enhanced energy efficiency mechanisms, resource virtualization, resource sharing, self-management and self-organization capabilities [8]. The concept of Future Networks envisages a broad range of opportunities in different fields. In other words, it will cover not only the traditional network fields but also other domains, such as e-health, energy efficiency, emergency services, public safety, IoT, machine-to-machine (M2M) communication, Information Centric Networking (ICN), among others. The applicability of SDN, NFV, SON and cloud computing opens the door to facilitate the deployment and management of services in an open business environment. Indeed, if we take into account technologies and advances of different initiatives, a possible architecture for future networks is proposed (Figure 4). On one hand, it presents a layered structure: infrastructure, virtualization, control and application layers, similar to the SDN approach. On the other hand, VNFs and NFV M\&O modules are incorporated in order to control the NFVI. For its part, cloud technologies are present on the cloud computing layer and SON capacities will aid in the decision process in the control layer.

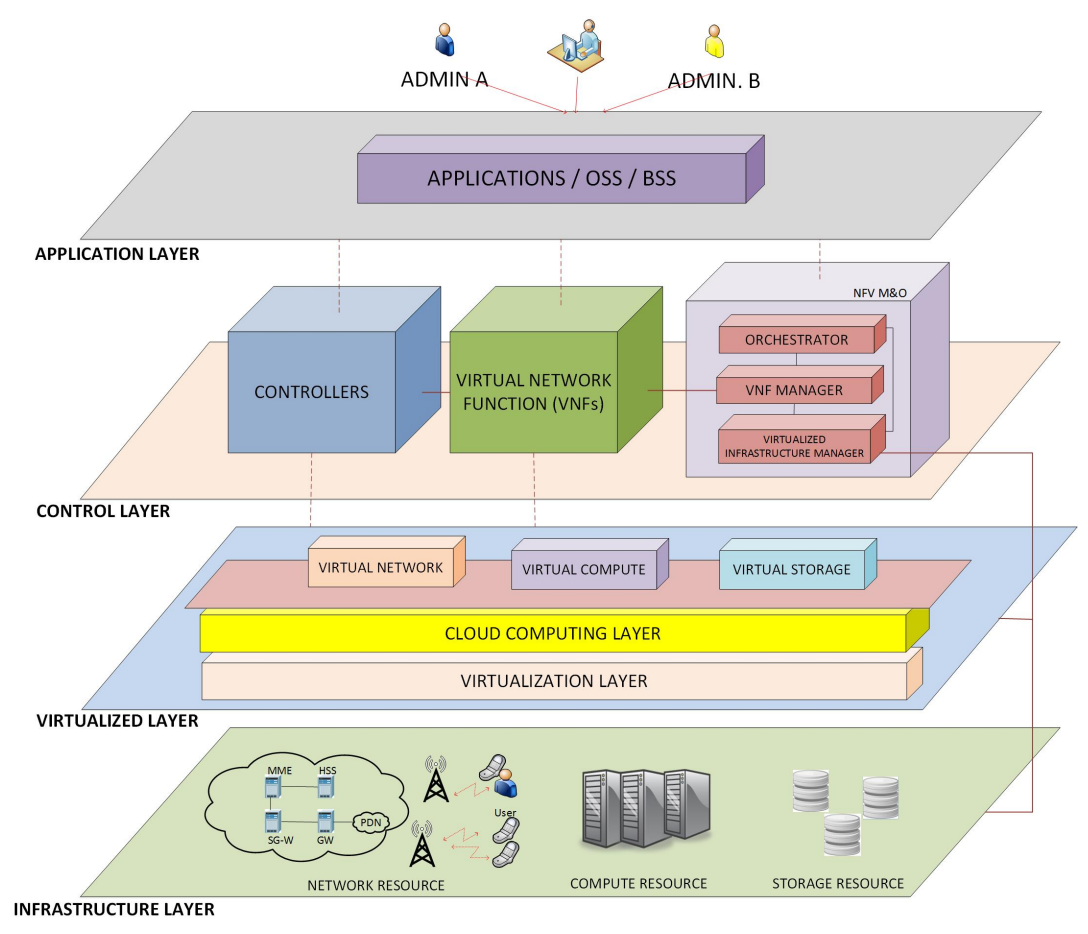

Figure 4. Future Network Architecture. 
Future Networks could incorporate all of these concepts or part of them. Despite of the advantages of this proposal, there are some challenges that need to be overcome in order to successfully combine these technologies. Firstly, the unified definition and standardization in the separation of the data plane and control plane and the provision of virtualized instances will enable the easy development and integration of the future network technologies. In addition, the complexity of the mobile network elements constitutes a big challenge by itself. At the same time, these kind of systems will require effective pricing schemes and business models with two objectives in mind: (i) Customers pay only for the provisioned service and (ii) stakeholders receive revenues according to their SLAs. Another important issue is how legacy networks will coexist with new systems, which is still a relatively unexplored field.

In the management and orchestration field, significant changes are required not only to improve the processing of data information but also to optimize the deployment and allocation of network resources. A unified management framework could allow enhanced traffic monitoring, provide self-management capabilities and network customization. A virtualized environment faces some issues, such as finding the best place to allocate virtual functions (operator infrastructure or cloud), migration and scheduling process. Mechanisms are also needed to provide load balancing, energy efficiency algorithms, inter-domain capabilities, among others. In parallel, all of these characteristics should be provided in a secure and trusted environment with enhanced capacities to recovery from failures. Moreover, The SDN centralized control or the dynamism of cloud computing are challenges that need to be covered. Table 2 shows the challenges and future trends that must be covered in order to fulfill the user needs of $5 \mathrm{G}$ networks.

Table 2. Current Trends and Challenges.

\begin{tabular}{|c|c|c|}
\hline Requirement & Challenge & Future Trends/Enabler Technologies \\
\hline System Performance & $\begin{array}{l}\text { - Provide efficient mechanisms } \\
\text { regarding to radio } \\
\text { resource provisioning. } \\
\text { - Improving the capacity of } \\
\text { radio resources. } \\
\text { - Provide super wide bandwidth. } \\
\text { - Better management of data traffic, } \\
\text { interference and mobility levels. }\end{array}$ & $\begin{array}{l}\text { - Evolution of radio-access } \\
\text { technologies (RATs). } \\
\text { - Decreasing the cell size. } \\
\text { - Millimeter-wave communication } \\
\text { - Intelligent resource allocation via } \\
\text { SDN or SON. }\end{array}$ \\
\hline Composite Wireless Infrastructures & $\begin{array}{l}\text { The } 5 \mathrm{G} \text { device can choose the most } \\
\text { appropriate wireless or mobile } \\
\text { technology according their needs } \\
\text { (Change between systems). }\end{array}$ & $\begin{array}{l}\text { - Enhancement of user devices } \\
\text { (Muti-Band-Multi-Mode support). } \\
\text { - Introduction of intelligent } \\
\text { mechanisms and SDN control. }\end{array}$ \\
\hline $\begin{array}{l}\text { Facilitating very dense } \\
\text { deployments (Hetnets) }\end{array}$ & $\begin{array}{l}\text { Operators must provide effective } \\
\text { mechanisms to deploy cells of different } \\
\text { sizes according to user needs. }\end{array}$ & $\begin{array}{l}\text { - Improving the resource capacity } \\
\text { through decreasing the cell size. } \\
\text { - Introduction of intelligent and } \\
\text { Software Defined Radio (SDR) concepts. }\end{array}$ \\
\hline Flexible spectrum management & $\begin{array}{l}\text { Improve the spectrum utilization in } \\
\text { order to operate in some spectrum } \\
\text { bands or channels, while } \\
\text { reducing interferences. }\end{array}$ & $\begin{array}{l}\text { - Massive MIMO } \\
\text { - Mechanisms to use unused bands. }\end{array}$ \\
\hline $\begin{array}{l}\text { Native support D2D } \\
\text { Communication }\end{array}$ & $\begin{array}{l}\text { Deploy networks based on } \\
\text { interconnected end user devices } \\
\text { (machines, sensors, etc). The traffic will } \\
\text { be properly assigned without } \\
\text { cause congestions. }\end{array}$ & $\begin{array}{l}\text { - Introduction of Cognitive Intelligent } \\
\text { mechanisms to exchange traffic } \\
\text { between users. } \\
\text { - Smarter end-user devices. }\end{array}$ \\
\hline Reduce Capex and Opex & $\begin{array}{l}\text { - Reduce the average service creation. } \\
\text { - Dynamic scalability and } \\
\text { deployment of services and NFs, while } \\
\text { reducing the complexity in planning } \\
\text { and configuration tasks. }\end{array}$ & $\begin{array}{l}\text { - Resource sharing (Exploring } \\
\text { Cloud-RAN, Cloud computing, NFV) } \\
\text { - Smarter allocation of functional } \\
\text { mobile components (SDN, NFV). }\end{array}$ \\
\hline
\end{tabular}


Table 2. Cont.

\begin{tabular}{|c|c|c|}
\hline Requirement & Challenge & Future Trends/Enabler Technologies \\
\hline $\begin{array}{l}\text { Muti-tenancy and } \\
\text { multi-service support }\end{array}$ & $\begin{array}{l}\text { Service providers can control the } \\
\text { resources deployed in a shared } \\
\text { infrastructure (network, computing, } \\
\text { mobile resources). }\end{array}$ & $\begin{array}{l}\text { - Cloud computing } \\
\text { - SDN } \\
\text { - NFV } \\
\text { - Mobile Edge Computing (MEC) }\end{array}$ \\
\hline Open Environment & $\begin{array}{l}\text { New applications and NFs could be } \\
\text { deployed in an open environment, } \\
\text { regardless of the network hardware and } \\
\text { technologies used by operators. }\end{array}$ & $\begin{array}{l}\text { - Standardization of SDN and } \\
\text { NFV concepts. } \\
\text { - Introduction of SDR. }\end{array}$ \\
\hline Energy efficiency operation & $\begin{array}{l}\text { Saving energy per service provided. } \\
\text { Nowadays, most of the energy } \\
\text { consumption comes from } \\
\text { RAN elements. }\end{array}$ & $\begin{array}{l}\text { Introduction of intelligent and SON } \\
\text { capabilities taking into account the } \\
\text { device status. }\end{array}$ \\
\hline Monitoring and Management & $\begin{array}{l}\text { Provide self-management and } \\
\text { self-optimization capabilities to } \\
5 G \text { systems. }\end{array}$ & $\begin{array}{l}\text { - Automated management and } \\
\text { monitoring functions (SDN, NFV). } \\
\text { - Takes decisions based on historical } \\
\text { record of network status. }\end{array}$ \\
\hline Ensuring QoS/ QoE and SLA & $\begin{array}{l}\text { A } 5 G \text { user will be able to obtain } \\
\text { enhanced services, regardless of the } \\
\text { location or network technologies } \\
\text { (compared with } 4 \mathrm{G} \text { systems), for } \\
\text { several use cases such as emergency } \\
\text { situations or network failures. }\end{array}$ & $\begin{array}{l}\text { - Enhanced mechanism to monitor } \\
\text { the network status (traffic optimization } \\
\text { techniques) via SDN and } \\
\text { intelligent mechanism. } \\
\text { - Automated network configuration } \\
\text { to ensure the required need } \\
\text { (SDN, NFV). }\end{array}$ \\
\hline Charging and billing & $\begin{array}{l}\text { Create different user profiles so that } \\
\text { customers pay only the required service } \\
\text { (pay-as-you-go), while operators bill } \\
\text { the respective service. }\end{array}$ & Introduction of SDN and NFV concepts. \\
\hline
\end{tabular}

It is important to note the current efforts of initiatives such as 5G Americas [46], 5G-PPP or NGMN to develop future network. They promote not only SDN, NFV and cloud computing adoption but also the study of transversal concepts such as carrier aggregation, massive MIMO, Multi-RAT convergence, spectral and signaling efficiency, among others. It is imperative that telecommunication and network service providers find a consensus to develop solutions, architectures, technologies and standards for the next generation of infrastructures. The communication paradigm of anytime, anyhow and anywhere will become a reality in the future society.

\section{Conclusions}

The next generation of mobile networks will be able to support higher capacity, lower latency and massive network access compared with current mobile deployments. Future mobile systems require the enhancement of radio and network elements, which takes advantage of intelligent mechanisms, cloud computing, SDN and NFV approaches. At the same time, these technologies foster the service innovation and provide ubiquitous and on-demand features. This paper has discussed SDN, NFV, Cloud Computing and SON concepts as enabler technologies to design future mobile networks. We have focused on concepts that could aid to cover operational and management requirements as well as radio and network capabilities. It also presents the ongoing projects and use cases that leverage these technologies in the context of mobile networks. This work is intended to explain the benefits that we could obtain from the combination between these concepts and the challenges to lay the foundations of Future Mobile Networks.

Acknowledgments: This work is supported by the European Commission Horizon 2020 Programme under grant agreement number H2020-ICT-2014-2/671672 - SELFNET (Framework for Self-Organized Network Management 
in Virtualized and Software Defined Networks). Lorena Isabel Barona López and Ángel Leonardo Valdivieso Caraguay are supported by the Secretaría Nacional de Educación Superior, Ciencia, Tecnología e Innovación SENESCYT (Quito, Ecuador) under Convocatoria Abierta 2012 and 2013 Scholarship Program.

Author Contributions: The authors contributed equally to this research.

Conflicts of Interest: The authors declare no conflict of interest.

\section{References}

1. European Commission. Future Internet 2020: Visions of an Industry Expert Group 2009. Available online: http://www.future-internet.eu/fileadmin/documents/reports/FI_Panel_Report_v3.1_Final.pdf (accessed on 16 December 2016).

2. Nokia Network. 5G Use Cases \& Requirements. 2014. Available online: http://networks.nokia.com/file/ 31121/5g-requirements (accessed on 16 December 2016).

3. Agyapong, P.K.; Iwamura, M.; Staehle, D.; Kiess, W.; Benjebbour, A. Design Considerations for a 5G Network Architecture. IEEE Commun. Mag. 2014, 52, 65-75.

4. NGMN Alliance. NMGN 5G White Paper 2015. Available online: https://www.ngmn.org/uploads/media/ NGMN_5G_White_Paper_V1_0.pdf (accessed on 16 December 2016).

5. Boccardi, F.; Heath, R.W.; Lozano, A.; Marzetta, T.L.; Popovski, P. Five Disruptive Technology directions for 5G. IEEE Commun. Mag. 2014, 52, 74-80.

6. Gupta, A.; Jha, R.K. A Survey of 5G Network: Architecture and Emerging Technologies. IEEE Access 2015, 3, 1206-1232.

7. Bangerter, B.; Talwar, S.; Arefi, R.; Stewart, K. Networks and Devices for the 5G Era. IEEE Commun. Mag. 2014, 52, 90-96.

8. Andrews, J.G.; Buzzi, S.; Choi, W.; Hanly, S.V.; Lozano, A.; Soong, A.C.; Zhang, J.C. What Will 5G Be? IEEE J. Sel. Areas Commun. 2014, 32, 1065-1082.

9. Demestichas, P.; Georgakopoulos, A.; Karvounas, D.; Tsagkaris, K.; Stavroulaki, V.; Lu, J.; Xiong, C.; Yao, J. 5G on the Horizon: Key Challenges for the Radio-Access Network. IEEE Veh. Technol. Mag. 2013, 8, 47-53.

10. Kreutz, D.; Ramos, F.M.V.; Verissimo, P.E.; Rothenberg, C.E.; Azodolmolky, S.; Uhlig, S. Software-defined Networking: A Comprehensive Survey. Proc. IEEE 2015, 103, 14-76.

11. ETSI Industry Specification Group (ISG). Network Function Virtualization (NFV) Architectural Framework. 2013. Available online: http://www.etsi.org/technologies-clusters/technologies/nfv (accessed on 16 December 2016).

12. Zhang, Q.; Cheng, L.; Boutaba, R. Cloud Computing: State-of-the-art and Research Challenges. J. Int. Ser. Appl. 2010, 1, 7-18.

13. Baldo, N.; Giupponi, L.; Mangues-Bafalluy, J. Big Data Empowered Self Organized Networks. In Proceedings of the 20th European Wireless Conference, Barcelona, Spain, 14-16 May 2014.

14. Buda, T.S.; Assem, H.; Xu, L.; Raz, D.; Margolin, U.; Rosensweig, E.; Lopez, D.R.; Corici, M.I.; Smirnov, M.; Mullins, R.; et al. Can Machine Learning aid in delivering new Use Cases and Scenarios in 5G? IEEE Netw. Oper. Manag. Symp. (NOMS) 2016, 1279-1284, doi:10.1109/NOMS.2016.7503003.

15. Mijumbi, R.; Serrat, J.; Gorricho, J.L.; Bouten, N.; Turck, F.; Boutaba, R. Network Function Virtualization: State-of-the-art and Research Challenges. IEEE Commun. Surv. Tutor. 2015, 18, 236-262.

16. Contreras, L.M.; Doolan, P.; Lonsethagen, H.; López, R. Operational, Organizational and Business Challenges for Network Operators in the context of SDN and NFV. Comput. Netw. 2015, 92, 211-217.

17. Hu, F.; Hao, Q.; Bao, K. A Survey on Software-Defined Network and OpenFlow: From Concept to Implementation. IEEE Commun. Surv. Tutor. 2014, 16, 1-27.

18. McKeown, N.; Anderson, T.; Balakrishnan, H.; Parulkar, G.; Peterson, L.; Rexford, J.; Shenker, S.; Turner, J. OpenFlow: Enabling Innovation in Campus Networks. ACM SIGCOMM Comput. Commun. Rev. 2008, 38, 69-74.

19. Sefraoui, O.; Aissaoui, M.; Eleuldj, M. OpenStack: Toward an Open-source Solution for Cloud Computing. Int. J. Comput. Appl. 2012, 55, 38-42.

20. Sonkoly, B.; Németh, F.; Csikor, L.; Gulyás, L.; Gulyás, A. SDN Based Testbeds for Evaluating and Promoting Multipath TCP. In Proceedings of the 2014 IEEE International Conference on Communications (ICC), Sydney, Australia, 10-14 June 2014; pp. 3044-3050. 
21. Pentikousis, K.; Wang, Y.; Hu, W. MobileFlow: Toward Software-defined Mobile Networks. IEEE Commun. Mag. 2013, 51, 44-53.

22. EU CROWD Project. Connectivity Management for EneRgy Optimised Wireless Dense Networks. Project Reference: 318115. Funded under: FP7-ICT. Available online: http://www.ict-crowd.eu/ (accessed on 16 December 2016).

23. Abdelwahab, S.; Hamdaoui, B.; Guizani, M.; Znati, T. Network Function Virtualization in 5G. IEEE Commun. Mag. 2016, 54, 84-91.

24. Open Networking Foundation (ONF), 2016. Available online: https://www.opennetworking.org/ (accessed on 16 December 2016).

25. ETSI Industry Specification Group (ISG). Network Function Virtualization Use Cases 2013. Available online: http://www.etsi.org/deliver/etsi_gs/nfv/001_099/001/01.01.01_60/gs_nfv001v010101p.pdf (accessed on 16 December 2016).

26. Open Platform for NFV (OPNFV). Available online: https://www.opnfv.org/ (accessed on 16 December 2016).

27. Floodlight SDN Controller. Available online: https://github.com/floodlight/floodlight (accessed on 16 December 2016).

28. OpenDaylight SDN Controller. Available online: https://www.opendaylight.org/start (accessed on 16 December 2016).

29. 5G Infrastructure Public Private Partnership - 5G PPP. Available online: https://5g-ppp.eu (accessed on 16 December 2016).

30. IMT-2020 (5G) Promotion Group. Available online: http://www.imt-2020.cn/en/introduction (accessed on 16 December 2016).

31. 5G Forum. Available online: http:/ /www.5gforum.org/ (accessed on 16 December 2016).

32. Fifth Generation Mobile Communication Promotion Forum (5GMF). Available online: http://5gmf.jp/en/ (accessed on 16 December 2016).

33. Osseiran, A.; Boccardi, F.; Braun, V.; Kusume, K.; Marsch, P.; Maternia, M.; Queseth, O.; Schellmann, M.; Schotten, M.; Taoka, H.; et al. Scenarios for 5G Mobile and Wireless Communications: The Vision of the METIS Project. IEEE Commun. Mag. 2014, 52, 26-35.

34. MCN Project. Funded under: FP7-ICT. Project Reference: 318109, Funded under: FP7-ICT. Available online: http:/ / www.mobile-cloud-networking.eu/site/ (accessed on 16 December 2016).

35. CONTENT Project. Convergence of Wireless Optical Network and iT rEsources iN SupporT of Cloud Services. FP7-ICT. Project Reference: 318514, Funded under: FP7-ICT. Available online: http:/ / cordis.europa.eu/fp7/ict/future-networks/documents/call8-projects/content-factsheet.pdf (accessed on 16 December 2016).

36. EU T-NOVA Project. Network Functions as-a-Service over Virtualised Infrastructures. Project Reference: 619520. Funded under: FP7-ICT. Available online: http:/ /www.t-nova.eu/ (accessed on 16 December 2016).

37. EU UNIFY Project. Unifying Cloud and Carrier Networks. Project Reference: 619609. Funded under: FP7-ICT. Available online: http:/ /www.fp7-unify.eu/ (accessed on 16 December 2016).

38. 5G-NORMA Project. 5G NOvel Radio Multiservice Adaptive Network Architecture. Project Reference: 671584. Funded under: H2020-ICT-2014-2. Available online: https://5gnorma.5g-ppp.eu/ (accessed on 16 December 2016).

39. METIS-II Project. Mobile and Wireless Communications Enablers for Twenty-Twenty (2020) Information Society-II. Project Reference: 671680. Funded under: H2020-ICT-2014-2. Available online: https://5g-ppp. eu/metis-ii/ (accessed on 16 December 2016).

40. CHARISMA Project. Converged Heterogeneous Advanced 5G Cloud-RAN Architecture for Intelligent and Secure Media Access. Project Reference: 671704. Funded under: H2020-ICT-2014-2. Available online: http:/ / www.charisma5g.eu// (accessed on 16 December 2016).

41. SONATA Project. Service Programing and Orchestration for Virtualized Software Networks. Project Reference: 671517. Funded under: H2020-ICT-2014-2. Available online: http://www.sonata-nfv.eu// (accessed on 16 December 2016).

42. Flex5Gware Project. Flexible and Efficient Hardware/Software Platforms for 5G Network Elements and Devices. Project Reference: 671563. Funded under: H2020-ICT-2014-2. Available online: http://www. flex5gware.eu/ (accessed on 16 December 2016). 
43. Neves, P.; Calé, R.; Costa, M.R.; Parada, C.; Parreira, B.; Alcaraz-Calero, J.; Wang, Q.; Nightingale, J.; Chirivella-Perez, E.; Jiang, W.; et al. The SELFNET Approach for Autonomic Management in an NFV/SDN Networking Paradigm. Int. J. Distrib. Sens. Net. 2016, 2016, 1-17.

44. SELFNET Project. Framework for Self-Organized Network Management in Virtualized and Software Defined Networks. Project reference: 671672. Funded under: H2020-ICT-2014-2. Available online: https:/ / selfnet-5g.eu/ (accessed on 16 December 2016).

45. Xu, L.; Assem, H.; Yahia, I.G.B.; Buda, T.S.; Martin, A.; Gallico, D.; Biancani, M.; Pastor, A.; Aranda, P.A.; Smirnov, M.; et al. CogNet: A Network Management Architecture Featuring Cognitive Capabilities. IEEE Netw. Commun. (EuCNC) 2016, 2016, 325-329.

46. 5G Americas, 2016. Available online: http://www.5gamericas.org/es/ (accessed on 16 December 2016).

(C) 2016 by the authors; licensee MDPI, Basel, Switzerland. This article is an open access article distributed under the terms and conditions of the Creative Commons Attribution (CC-BY) license (http://creativecommons.org/licenses/by/4.0/). 